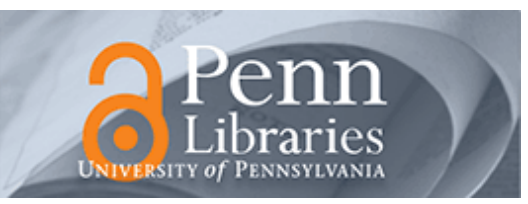

University of Pennsylvania

ScholarlyCommons

\title{
Cooperative profit sharing in coalition based resource allocation in wireless networks
}

\author{
Alireza Aram \\ University of Pennsylvania, aaram@seas.upenn.edu \\ Chandramani Singh \\ Indian Institute of Science \\ Saswati Sarkar \\ University of Pennsylvania, swati@seas.upenn.edu \\ Anurag Kumar \\ Indian Institute of Science \\ Follow this and additional works at: https://repository.upenn.edu/ese_papers \\ Part of the Electrical and Computer Engineering Commons
}

\section{Recommended Citation}

Alireza Aram, Chandramani Singh, Saswati Sarkar, and Anurag Kumar, "Cooperative profit sharing in coalition based resource allocation in wireless networks", . April 2009.

Copyright 2009 IEEE. Reprinted from:

Aram, A.; Singh, C.; Sarkar, S.; Kumar, A., "Cooperative Profit Sharing in Coalition Based Resource Allocation in Wireless Networks," INFOCOM 2009, IEEE , vol., no., pp.2123-2131, 19-25 April 2009

URL: http://ieeexplore.ieee.org/stamp/stamp.jsp?arnumber=5062136\&isnumber=5061888

This material is posted here with permission of the IEEE. Such permission of the IEEE does not in any way imply IEEE endorsement of any of the University of Pennsylvania's products or services. Internal or personal use of this material is permitted. However, permission to reprint/republish this material for advertising or promotional purposes or for creating new collective works for resale or redistribution must be obtained from the IEEE by writing to pubs-permissions@ieee.org. By choosing to view this document, you agree to all provisions of the copyright laws protecting it.

This paper is posted at ScholarlyCommons. https://repository.upenn.edu/ese_papers/515

For more information, please contact repository@pobox.upenn.edu. 


\title{
Cooperative profit sharing in coalition based resource allocation in wireless networks
}

\begin{abstract}
We consider a network in which several service providers offer wireless access service to their respective subscribed customers through potentially multi-hop routes. If providers cooperate, i.e., pool their resources, such as spectrum and base stations, and agree to serve each others' customers, their aggregate payoffs, and individual shares, can potentially substantially increase through efficient utilization of resources and statistical multiplexing. The potential of such cooperation can however be realized only if each provider intelligently determines who it would cooperate with, when it would cooperate, and how it would share its resources during such cooperation. Also, when the providers share their aggregate revenues, developing a rational basis for such sharing is imperative for the stability of the coalitions. We model such cooperation using transferable payoff coalitional game theory. We first consider the scenario that locations of the base stations and the channels that each provider can use have already been decided apriori. We show that the optimum cooperation strategy, which involves the allocations of the channels and the base stations to mobile customers, can be obtained as solutions of convex optimizations. We next show that the grand coalition is stable in this case, i.e. if all providers cooperate, there is always an operating point that maximizes the providers' aggregate payoff, while offering each such a share that removes any incentive to split from the coalition. Next, we show that when the providers can choose the locations of their base stations and decide which channels to acquire, the above results hold in important special cases. Finally, we examine cooperation when providers do not share their payoffs, but still share their resources so as to enhance individual payoffs. We show that the grand coalition continues to be stable.
\end{abstract}

\section{Keywords}

channel allocation, game theory, radio networks, resource allocation, channel allocations, coalition stability, convex optimizations, cooperative profit sharing, game theory, mobile customers, multihop routes, wireless access service, wireless networks resource allocation

\section{Disciplines}

Electrical and Computer Engineering

\section{Comments}

Copyright 2009 IEEE. Reprinted from:

Aram, A.; Singh, C.; Sarkar, S.; Kumar, A., "Cooperative Profit Sharing in Coalition Based Resource Allocation in Wireless Networks," INFOCOM 2009, IEEE , vol., no., pp.2123-2131, 19-25 April 2009

URL: http://ieeexplore.ieee.org/stamp/stamp.jsp?arnumber=5062136\&isnumber=5061888

This material is posted here with permission of the IEEE. Such permission of the IEEE does not in any way imply IEEE endorsement of any of the University of Pennsylvania's products or services. Internal or personal use of this material is permitted. However, permission to reprint/republish this material for advertising or promotional purposes or for creating new collective works for resale or redistribution must be obtained from the IEEE by writing to pubs-permissions@ieee.org. By choosing to view this document, you agree to all provisions of the copyright laws protecting it. 


\title{
Cooperative Profit Sharing in Coalition Based Resource Allocation in Wireless Networks
}

\author{
Alireza Aram* ,Chandramani Singh, Saswati Sarkar, Anurag Kumar
}

\begin{abstract}
We consider a network in which several service providers offer wireless access service to their respective subscribed customers through potentially multi-hop routes. If providers cooperate, i.e., pool their resources, such as spectrum and base stations, and agree to serve each others' customers, their aggregate payoffs, and individual shares, can potentially substantially increase through efficient utilization of resources and statistical multiplexing. The potential of such cooperation can however be realized only if each provider intelligently determines who it would cooperate with, when it would cooperate, and how it would share its resources during such cooperation. Also, when the providers share their aggregate revenues, developing a rational basis for such sharing is imperative for the stability of the coalitions. We model such cooperation using transferable payoff coalitional game theory. We first consider the scenario that locations of the base stations and the channels that each provider can use have already been decided apriori. We show that the optimum cooperation strategy, which involves the allocations of the channels and the base stations to mobile customers, can be obtained as solutions of convex optimizations. We next show that the grand coalition is stable in this case, i.e. if all providers cooperate, there is always an operating point that maximizes the providers' aggregate payoff, while offering each such a share that removes any incentive to split from the coalition. Next, we show that when the providers can choose the locations of their base stations and decide which channels to acquire, the above results hold in important special cases. Finally, we examine cooperation when providers do not share their payoffs, but still share their resources so as to enhance individual payoffs. We show that the grand coalition continues to be stable.
\end{abstract}

\section{INTRODUCTION}

We have witnessed a significant growth in commercial wireless services in the past few years, and the trend is likely to continue in the foreseeable future. Satisfaction of this increasing demand is contingent upon efficient utilization of the transmission resources, which are either limited (e.g., spectrum), or costly (e.g. infrastructure). Currently, most of the available resources are substantially under-utilized, e.g., utilization of licensed spectrum is at times only $15 \%$ ! ( [1]).

Cooperation among different wireless providers has the potential for substantially improving the utilization of the available resources, and should therefore enhance the proliferation of wireless services. In particular, different providers may form a coalition and pool their resources, such as spectrum and infrastructure like base stations and relay nodes, and serve each others' customers. Such coalitions may lead to substantially higher throughput through statistical multiplexing and lower overall energy consumptions of the customers through multihop relaying. Both of these in turn lead to higher customer satisfaction, and higher payoff for the providers. Cooperation

*A. Aram \& S. Sarkar are in the Dep. of Electrical and Systems Eng., University of Pennsylvania. Their contributions have bean supported by NSF grants NCR- 0238340, CNS-0721308, ECS-0622176. C. Singh \& A. Kumar are in the Electrical Communications Eng., Indian Institute of Science. may also be instrumental in reducing the costs incurred by the providers in acquiring spectrum and deploying infrastructure like base stations. This would again lead to higher net payoff for the providers. We now elucidate the above benefits using a sequence of examples.

We first demonstrate how cooperation may substantially enhance throughput and decrease energy consumption of customers. Transmission qualities of available channels randomly fluctuate with time and space, owing to customer mobility and propagation conditions. Also, in secondary access networks, the providers may be secondary users who do not license channels but communicate when the license holders (primary users) do not use the channels. Such access opportunities may only arise sporadically. Since all customers of all providers do not need to be served simultaneously, and the channels of different providers may not be unavailable or have poor transmission qualities simultaneously, spectrum pooling can mitigate service fluctuations caused by occasional variations in channel qualities and availabilities, and instantaneous traffic overloads. This enhances throughput and delivers stringent service qualities demanded by the customers. In multi-hop wireless networks (e.g., mesh networks), cooperation increases the number of available relays (mesh points). This in turn increases the number of multi-hop routes to each customer. Thus, the total power usage of the customers could significantly decrease, and total throughput may significantly increase. In addition, the customers may be induced to serve as relays, perhaps, in lieu of service discounts. Then the enhancement in throughput and energy consumption owing to cooperation magnifies as the coalitions have a larger set of customers, and therefore a larger number of multi-hop routes.

We now demonstrate how cooperation may substantially reduce the costs incurred by the providers. A provider can acquire a channel by paying a fixed licensing cost or usage based charges, or a combination of the two. The first case arises when the providers are primary users who license the channels from government agencies, and the other options arise when they are secondary users who use the channels licensed by the primaries. When the providers do not cooperate, they may need to operate as secondary users and opt primarily for usage based charges, as the volume of their individual traffic may not justify other options. Since cooperation allows the providers to pool the customers, the resulting higher aggregate traffic may allow them to license channels, share the licensing fees and thereby reduce the individual costs. Next, deploying new base stations (access points) and subsequently maintaining them, is one of the major costs in expanding the networks. Cooperation may allow the individual providers to deliver desired coverage and throughput guarantees to their customers while deploying fewer base stations. We demonstrate this advantage using a 
specific scenario which arises in practice. Consider a provider whose customer base is concentrated in a particular region. Traffic demand is therefore high in this region and low, but non-zero, in other regions. This low traffic demand in other regions is generated primarily because of the mobility of its customers. In order to provide universal coverage to its customers (otherwise they would desert), the provider must deploy base stations throughout, i.e., even in the regions where its traffic demand is low. These base stations would however be used very little. If instead, the provider cooperates with another whose traffic demand is concentrated in a different region, both may satisfy coverage requirements by deploying base stations only in the regions where their individual demands are concentrated. This would in turn significantly reduce the expenses incurred by each. Such cooperation would also allow the providers to expand their networks without deploying new base stations in the regions where they are expanding to.

Several research challenges must however be addressed before large scale cooperation can be realized. First, commercial service providers are selfish entities who seek to maximize their individual payoffs. Therefore, they will cooperate with others only when this increases their individual incomes. Even so, a provider is likely to refuse to join a coalition if it perceives that its share of the aggregate payoff is not proportional to the amount it invested and the wealth it generated. The former depends on the transmission rates in the channels it has acquired and the locations and the number of base stations it has deployed. The latter depends on its customer base. So, developing a rational basis for determining the individual shares of the aggregate payoff is imperative. Note that the aggregate payoff and the individual shares depend on the cooperation strategies of the providers. Specifically, each provider needs to decide which providers it would cooperate with, which channels it is going to use, the locations where it is going to construct new base stations, and when it should serve the customers of another provider. The sharing mechanism and the optimal cooperation strategies for each provider depend on each other and must be obtained jointly.

We present a framework to determine the optimal decisions of the providers using tools from cooperative game theory. The framework also provides a rational basis for sharing the aggregate payoff. Using tools from transferable payoff coalitional game theory, we first develop this framework assuming that the providers can share the aggregate payoff in any manner they wish to. The first network setup we consider is an access network where providers pool their spectrum, base stations and customers. We assume that the locations of the base stations and the set of channels they have access to, are determined apriori, but the providers decide how they would allocate the base stations and the channels of the coalition, to the customers. We then obtain optimal decision rules for the providers and a strategy for sharing the resulting aggregate payoff as solutions of concave optimization problems. This sharing strategy ensures that it is optimal for all providers to cooperate (section III). Specifically, if any subset of providers split from the grand coalition (the coalition of all providers), irrespective of how they cooperate and the way they share their aggregate payoff, at least one provider in this subset will receive less net payoff than when it was in the grand coalition. In coalitional game terminology, such a sharing scheme exists only when the core of the game is nonempty. This result is of interest in itself as many cooperative games have empty cores, and the specific games we consider do not satisfy some standard sufficiency conditions for non-emptiness of the core (e.g., convexity of the game). We then consider the case where in addition, the providers need to determine the locations and numbers of base stations, and the set of channels for each base station. We obtain the optimal decision rules and the payoff sharing mechanism in some important special cases of this general problem (section IV). Subsequently, we generalize the formulations and the results to include multihop transmissions. Finally, we consider a setting where the providers serve the customers by pooling their resources as before, but can not share the aggregate payoff in any manner they wish to. Now, each can only claim the payoff it generates, i.e., the payoff fetched by its customers minus the cost it incurs to acquire channels and deploy its base stations. Note that cooperation may still increase the individual earnings as the customers are served using the aggregated resources. We formulate this problem as a nontransferable payoff coalition game problem (section VI) and show that the grand coalition is still stable. That is, there always exists a joint action of the providers that makes it sub-optimal for any subset of them to leave the grand coalition.

\section{MODEL}

Consider a network with a set of providers $N$ and a set of customers. Each provider $i$ owns a set of customers $M_{i}$. In order to serve the customers, provider $i$ intends to open a number of base stations (access points) from a set of candidate locations $B_{i}$. Let $B_{i_{1}} \cap B_{i_{2}}=\emptyset$ and $M_{i_{1}} \cap M_{i_{2}}=\emptyset$ for $i_{1} \neq$ $i_{2}$. In figure 1 , for instance, $N=\{1,2\}, M_{1}=\{1\}$, and $B_{2}=$ $\{2,3\}$. Let $B_{N}$ and $M_{N}$ be the sets of candidate locations and customers in the system respectively. We assume that each provider uses its base stations to serve customers through a set of channels it has access to. Unless mentioned otherwise, base stations communicate with the customers through single-hop links. In order to get access to the channels, providers need to pay the corresponding charges. Each customer $j$ negotiates a minimum rate guarantee of $m_{j}$ with its provider apriori.

We assume that each base station $k$ can have access to a set of channels $C_{k}$. That is, base station $k$ is allowed to use any subset of channels in $C_{k}$ if the provider associated with $k$ pays the corresponding charges. For instance in figure 1, $C_{1}=\{1,4\}$. We also assume that no two base stations in a vicinity can have access to the same channel, i.e., $C_{k_{1}} \cap$ $C_{k_{2}}=\emptyset$ for $k_{1} \neq k_{2}$ if $k_{1}$ and $k_{2}$ are in the vicinity. Thus, the communications of different base stations with different customers do not interfere. So, channels can be numbered such that each channel belongs in only one $C_{k}$. In other words we can assume, without loss of generality, that $C_{k_{1}} \cap C_{k_{2}}=\emptyset$ for all $k_{1} \neq k_{2}$. For example, in figure 1 , channels 1 and 3 could be the same frequency band. But since base stations 1 and 3 are far apart, they can simultaneously use the same frequency band without interfering with each other. So we can assume that they are two different channels. Let $C_{N}$ be the set of all the numbered channels in the system. An important outcome 


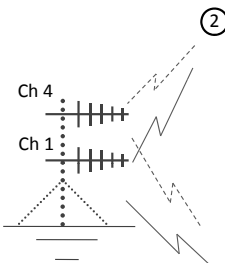

Base Station 1

(2)

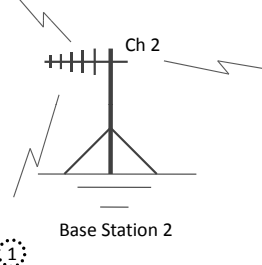

1

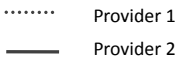

Fig. 1. An example of a network with two providers

of the numbering of channels in this way is that specifying a channel (e.g., l) uniquely determines its base station as well as provider.

The instantaneous rates the customers receive on different channels depend on the current quality of the channels (which in case of secondary access channels also includes the current actions of the channels' primary users) and the current positions of the customers, which can be random. We therefore assume that when customer $j$ is served by channel $l, j$ receives a rate $R_{l j}$, a random variable which is a function of the state of channel $l$ and position of customer $j$. Let $\Omega_{l j}$ be the state space of $R_{l j}$. We assume that $\left|\Omega_{l j}\right|$ is finite. This assumption is motivated by the fact that feasible service rates in any practical communication system belong to a finite set. Thus, we assume that each channel has a finite number of states. Also, we can partition the service region in such a way that the service rates received by the customers inside a member of the partition do not depend on the locations of the customers. Let $\Omega=\prod_{l \in C_{N}} \Omega_{l j}$ and $P(\omega)$ be the probability of an outcome $\omega \in \Omega$.

We now discuss the charging mechanisms used by spectrum regulators, which are government agencies or license holders (primary users). The charging mechanisms can be usage based, membership based, or a combination of these. As the names imply, membership charge is to be paid for a channel $l$ by a provider $i$, if it intends to use $l$ at some point in the operation, regardless of the amount of usage. Usage based charge for each channel depends only on the amount of usage and is 0 if the channel is not used. Also, different spectrum regulators may use different charging mechanisms, and different channels may provide different statistics for the transmission rates.

The expected payoff a provider earns depends on the rates it provides to its customers and the cost it incurs in using the channels. So, in order to maximize its payoff, a provider may need to judiciously decide (a) the set of channel to be used by each of its base stations, and (b) allocation of its customers to the base stations and the corresponding channels. Note that the allocations may vary with the rates available in these channels which in turn vary with time. Such payoffs are likely to increase if multiple providers cooperate, i.e., pool in their resources (base stations, channels) to serve the joint set of customers. We now propose a framework based on coalition game theory so as to capture the above interactions.

Definition II.1. A coalition $S \subseteq N$ is a subset of players (e.g., service providers) who cooperate. For a coalition $S, B_{S}$ and $M_{S}$ are the set of base station candidate locations and customers associated with providers in $S$, and $C_{S}=\cup_{k \in B_{S}} C_{k}$. We refer to $N$ as the grand coalition.

Definition II.2. A coalitional game with transferable payoff $\langle N, v\rangle$ consists of a finite set $N$ (set of providers) and a characteristic function $v($.$) that associates with every$ nonempty subset $S$ of $N$, a real number $v(S)$. For each coalition $S$, the number $v(S)$ is the aggregate payoff available for any arbitrary division among the members of $S$.

A channel $l \in C_{k}$ can serve customer $j$ only when both are associated with the same provider or the providers associated with them are in a coalition. Let random variable $\alpha_{l j} \in[0,1]$ be the fraction of time channel $l$ serves customer $j . \alpha_{l j} \mathrm{~s}$ are determined by the allocation scheme. When the provider associated with customer $j$ is in coalition $S$, the rate received by $j$ is a random variable $y_{j}(\omega)=\sum_{l \in C_{S}} \alpha_{l j}(\omega) R_{l j}(\omega)$. When customers associated with provider $i$ receive rates $Y_{i}(\omega)=\left\{y_{j}(\omega), j \in M_{i}\right\}$, they pay $i$ the amount $U_{i}\left(Y_{i}(\omega)\right)$, where $U_{i}($.$) is an increasing concave function and equal$ to 0 at the origin. Similarly, provider $i$ needs to pay the spectrum regulators a usage-based cost of $V_{i}\left(Z_{i}(\omega)\right)$, where $Z_{i}(\omega)=\left\{z_{l}(\omega), l \in C_{i}\right\}$ and $z_{l}(\omega)=\sum_{j \in M_{S}} \alpha_{l j}(\omega)$ is the total fraction of time channel $l$ is used. $V_{i}($.$) is an$ increasing convex function and equal to 0 at the origin. Then the total payoff available to a coalition $S$ will be the sum of the $U_{i}$ s for $i \in S$, reduced by $a$ ) the sum of the $V_{i} \mathrm{~s}$ for $l \in C_{S}$, and $b$ ) channels' membership-based charges and the costs of opening base stations. Therefore, providers in a coalition $S$ have to decide which base stations to open, which channels' memberships to buy, and how to schedule those base stations and channels to customers, based on their positions and utility and cost functions so as to maximize their total payoff, subject to possible minimum rate constraints. Now $v(S)$ is the maximum aggregate payoff available to a coalition $S$. We will show in the next sections how $v(S)$ can be obtained as a solution of an optimization problem. This optimization problem presents the optimum cooperation strategies among providers and optimal values for the decision variables. Note that the customers might also have maximum service rate constraints. This, however, is captured in our model, since the utility functions are concave and thus include upper-bounded functions.

Another important question now is how should these providers divide the aggregate payoff among each other. To answer this question, we introduce a solution concept in coalitional games known as the core. The idea behind the core in a cooperative game is analogous to that behind a Nash equilibrium of a noncooperative game: an outcome is stable if no deviation is profitable. 
Definition II.3. For any real valued vector $\boldsymbol{x}=$ $\left\{x_{1}, x_{2}, \ldots, x_{n}\right\}$ and any coalition $S$, we let $x(S)=\sum_{i \in S} x_{i}$. Such a vector is said to be an imputation if $x(N)=v(N)$ and $x_{i} \geq v(\{i\})$ for all $i \in N$. The core of the coalitional game with transferable payoff $\langle N, v\rangle$ is the set of all imputations $\boldsymbol{x}$ for which $x(S) \geq v(S)$ for all $S \subset N$. In other words

$$
\mathcal{C}=\left\{\mathbf{x} \in \mathbb{R}^{n}: \sum_{i \in N} x_{i}=v(N), \sum_{i \in S} x_{i} \geq v(S), \forall S \subset N\right\} .
$$

The significance of the core, comes from the fact that, every imputation in the core renders the grand coalition stable. To see this, let providers form the grand coalition and share $v(N)$ as per an imputation $\mathrm{x} \in \mathcal{C}$. Now, suppose a set of providers $S \subset$ $N$ split from the grand coalition and form a separate coalition to share their aggregate payoff $v(S)$ as per w. A provider $i \in$ $S$, however, would agree to split from the grand coalition only if $w_{i}>x_{i}$. This implies that $v(S)=\sum_{i \in S} w_{i}>\sum_{i \in S} x_{i}$, and thus contradicts the fact that $\mathrm{x} \in \mathcal{C}$. Therefore, the grand coalition is stable. This is a globally desirable outcome, since the grand coalition maximizes the aggregate payoff.

The core in several coalitional games is empty, i.e., the grand coalition cannot be stabilized, even if the grand coalition maximizes the aggregate payoff (Example 260.3 p. 260 [2]), and in general it is NP-hard to determine whether the core of a coalitional game is nonempty( [3]). Convexity ${ }^{1}$ of a game is a sufficient condition for the nonemptiness of the core. The games we consider, however, are not convex ( [4]). Nevertheless, in the following sections we show that the cores of the coalitional games for different network setups are nonempty, and we also obtain imputations in the respective cores.

\section{SPECTRUM POOLING GAME}

In this section, we formulate the cooperation among providers as a transferable payoff coalitional game and prove that it has a nonempty core. We assume that all providers have already decided where to open their base stations and which channels memberships to buy. In this scenario, the only decision variables to be determined are the scheduling of base stations and channels to customers. Thus, for simplicity, we assume that all base station opening and channel membership costs are zero. Consider the following convex optimization problem which returns $v(S)$ for any coalition $S \subseteq N$ if feasible, else $v(S)=-\infty$.

P(S):- Max: $\sum_{\substack{i \in S \\ \omega \in \Omega}} U_{i}\left(Y_{i}(\omega)\right) P(\omega)-V_{i}\left(Z_{i}(\omega)\right) P(\omega)$

\section{Subject to:}

1) $y_{j}(\omega)=\sum_{l \in C_{S}} \alpha_{l j}(\omega) R_{l j}(\omega), \quad j \in M_{S}, \omega \in \Omega$

2) $z_{l}(\omega)=\sum_{j \in M_{S}} \alpha_{l j}(\omega), \quad l \in C_{S}, \omega \in \Omega$

3) $\sum_{l \in C_{S}} \alpha_{l j}(\omega) \leq 1, \quad j \in M_{S}, \omega \in \Omega$

4) $\sum_{j \in M_{S}} \alpha_{l j}(\omega) \leq 1, \quad l \in C_{S}, \omega \in \Omega$

5) $\sum_{\omega \in \Omega} P(\omega) y_{j}(\omega) \geq m_{j}, \quad j \in M_{S}$

6) $\alpha_{l j}(\omega), y_{j}(\omega), z_{l}(\omega) \geq 0, \quad j \in M_{S}, l \in C_{S}, \omega \in \Omega$

Constraints (3) ensure that the total fraction of time each customer is served, is at most 1 . A channel can serve at most the whole fraction of time by (4). Constraints (5) guarantee

\footnotetext{
${ }^{1} \mathrm{~A}$ coalitional game is convex if $v(S)+v(T) \leq v(S \cup T)+v(S \cap T)$ for all $S, T \subseteq N$.
}

the minimum service rates. Note that the decision variables of the grand coalition can always be chosen as the union of the decision variables of the members of any partition of $N$. This means that the payoff of the grand coalition is at least equal to the sum of the payoffs of members of any partition.

The following example elucidates how cooperation can alter the optimal decision variables of providers ${ }^{2}$.

Example III.1. Consider the network in figure 1 with $N=$ $\{1,2\}$ and $B_{i}=C_{i}=\{i\}$ (we do not consider base station 3 or channel 3 and 4 here.). Let $R_{12}=R_{21}=R_{22}=P$ and $R_{11}=R_{23}=Q$, where $Q<P$, and $R_{l j}=0$ otherwise. Let $m_{j}=\frac{Q}{2}$ for $j=1 \ldots 3$. Suppose the payoffs equal the sum of the service rates. Then we have $v(\{1\})=Q$ and $v(\{2\})=$ $\frac{P+Q}{2}$. But if the two providers cooperate, with $\alpha_{11}=\alpha_{22}=0$, $\alpha_{12}=1$ and $\alpha_{21}=\alpha_{23}=\frac{1}{2}$, we have $v(\{1,2\})=\frac{3 P+Q}{2}$.

So, cooperation helps providers make a better use of their base stations, and as a result increases the aggregate payoff.

Using the duality technique, we now show that this game has a nonempty core. Let $\lambda, \beta \in \mathbb{R}^{\left|M_{S} \times \Omega\right|}$, $\nu, \gamma \in \mathbb{R}^{\left|C_{S} \times \Omega\right|}$, and $\rho \in \mathbb{R}^{\left|M_{S}\right|}$. Let $g_{i \omega}(\lambda, \rho)=$ $\max _{y_{j}(\omega) \geq 0}\left(P(\omega) U_{i}\left(Y_{i}(\omega)\right)+\sum_{j \in M_{i}} y_{j}(\omega)\left(\lambda_{j}(\omega)+\right.\right.$ $\left.\left.\rho_{j} P(\omega)\right)\right)$ and $h_{i \omega}(\nu)=\max _{z_{l}(\omega) \geq 0}\left(-P(\omega) V_{i}\left(Z_{i}(\omega)\right)+\right.$ $\left.\sum_{l \in C_{i}} z_{l}(\omega) \nu_{l}(\omega)\right)$. Then we have the following as the dual of $\mathrm{P}(S)$ :

D(S):- Minimize: $\sum_{i \in S}\left(\sum_{\omega \in \Omega}\left(g_{i \omega}+h_{i \omega}\right)+\sum_{\substack{l \in C_{i} \\ \omega \in \Omega}} \gamma_{l}(\omega)+\right.$ $\left.\sum_{j \in M_{i}} \beta_{j}(\omega)-\sum_{j \in M_{i}} m_{j} \rho_{j}\right)$

\section{Subject to:}
I) $\lambda_{j}(\omega) R_{l j}(\omega)+\nu_{l}(\omega)+\beta_{j}(\omega)+\gamma_{l}(\omega) \geq 0, l \in C_{S}, j \in$ $M_{S}, \omega \in \Omega$
II) $\beta_{j}(\omega), \gamma_{l}(\omega), \rho_{j} \geq 0, \quad l \in C_{S}, j \in M_{S}, \omega \in \Omega$

Formulate $\mathrm{D}(N)$ by appropriately defining vectors $\lambda, \beta, \gamma, \nu, \rho$ and let $\mathcal{D}$ constitutes the set of optimal solutions of $\mathrm{D}(N)$. Clearly, $\mathrm{D}(S)$ is feasible for each $S \subseteq N$. Thus, $\mathcal{D} \neq \emptyset$. Let

$$
\begin{aligned}
& \mathcal{I}=\left\{\mathbf{x}^{*} \in \mathbb{R}^{|N|}: x_{i}^{*}=\sum_{\omega \in \Omega}\left(g_{i \omega}\left(\lambda^{*}, \rho^{*}\right)+h_{i \omega}\left(\nu^{*}\right)\right)\right. \\
&+\sum_{\substack{j \in M_{i} \\
\omega \in \Omega}} \beta_{j}^{*}(\omega)-\sum_{j \in M_{i}} m_{j} \rho_{j}^{*}+\sum_{\substack{l \in C_{i} \\
\omega \in \Omega}} \gamma_{l}^{*}(\omega) \text { for some } \\
&\left.\left(\lambda^{*}, \nu^{*}, \beta^{*} \cdot \gamma^{*}, \rho^{*}\right) \in \mathcal{D}\right\}
\end{aligned}
$$

Here is the main result:

Theorem III.1. $\mathcal{I} \neq \emptyset$ and $\mathcal{I} \subseteq \mathcal{C}$.

Discussion: Note that $\mathrm{D}(\mathrm{S})$ is a convex optimization with linear constraints. Therefore, its optimal solution and thereby an imputation in the core can be computed in polynomial time [5]. In future, whenever we obtain an imputation in the core using the duality technique, the same argument follows.

Proof of theorem III.1: Since $\mathcal{D} \neq \emptyset, \mathcal{I} \neq \emptyset$. We show that for an arbitrary $\mathbf{x}^{*} \in \mathcal{I}, \mathbf{x}^{*} \in \mathcal{C}$. Note that, since $U_{i}$ s and $V_{i}$ s are (increasing) concave and convex functions respectively, the objective function of $\mathrm{P}(\mathrm{S})$ is concave. Also, the constraints

\footnotetext{
${ }^{2}$ In all the examples we assume stationary networks, i.e., $|\Omega|=1$. Thus all random quantities become deterministic.
} 
of $\mathrm{P}(\mathrm{S})$ are all linear. Therefore, $\mathrm{P}(\mathrm{S})$ is maximizing a concave function over a convex set. Thus, strong duality holds.

Now, consider an arbitrary $\mathbf{x}^{*} \in \mathcal{I}$, corresponding to one $\left(\lambda^{*}, \nu^{*}, \beta^{*} \cdot \gamma^{*}, \rho^{*}\right) \in \mathcal{D}$. Clearly $x^{*}(N)=\sum_{i \in N} x_{i}^{*}$ is the optimal value of $\mathrm{D}(N)$. Since $\mathrm{D}(S)$ is the dual of $\mathrm{P}(S)$ for each $S \subseteq N$, by strong duality $x^{*}(N)=v(N)$. Now we only need to show that $x^{*}(S) \geq v(S)$ for any $S \subset N$. If $\mathrm{P}(S)$ is infeasible, the claim is trivial for $v(S)=-\infty$. Suppose $\mathrm{P}(S)$ is feasible. By strong duality, $v(S)$ equals the optimum value of $\mathrm{D}(S)$. Consider the sub-vectors $\lambda_{S}^{*}, \nu_{S}^{*}, \beta_{S}^{*} \cdot \gamma_{S}^{*}, \rho_{S}^{*}$ consisting of the components of $\lambda^{*}, \nu^{*}, \beta^{*} \cdot \gamma^{*}, \rho^{*}$ in $S$. Clearly these sub-vectors constitute a feasible solution of $\mathrm{D}(S)$ and $x^{*}(S)$ is the value of the objective function of $\mathrm{D}(S)$ for the above feasible solution. Therefore, the optimal value of $\mathrm{D}(S)$ is a lower bound for $x^{*}(S)$, i.e., $x^{*}(S) \geq v(S)$.

Now we discuss how this framework can provide useful insights about the relation between a provider's payoff share, the resources it contributes, and the wealth it generates. Among the resources in possession of a provider, one could be more constrained than the others. For instance, a provider might have a lot of customers, but few base stations. Then, increasing the number of base stations could boost the payoff generated by the provider, while adding to the number of customers might not change it. The situation may be reversed for a different provider. Using the rule of thumb that more demand adds to the value of an asset, an intuitive observation then is that in a coalition, the provider that shares more of the resource that is sought most by the majority of the members of the coalition, is likely to receive a larger share of the aggregate payoff. The following example will further elucidate this.

Example III.2. Let $N=\{1,2,3\},\left|M_{1}\right|=5$, and $\left|M_{2}\right|=$ $\left|M_{3}\right|=2$. Also, let $\left|C_{1}\right|=2,\left|C_{2}\right|=3$, and $\left|C_{3}\right|=4$. Suppose $R_{l j}=P$ for all $l \in C_{N}$ and $j \in M_{N}$. Let the payoffs be equal to sum of the customers' service rates. It is easy to check that, $v(\{i\})=2 P$ for $i \in N, v(\{1,2\})=5 P$, $v(\{1,3\})=6 P, v(\{2,3\})=4 P$, and $v(\{1,2,3\})=9 P$. Thus, $\mathcal{C}=\left\{\left(x_{1}, x_{2}, x_{3}\right): x_{1}+x_{2}+x_{3}=9 P, x_{i} \geq 2 P, x_{1}+\right.$ $\left.x_{2} \geq 5 P, x_{1}+x_{3} \geq 6 P, x_{2}+x_{3} \geq 4 p\right\}$. Note that provider 1 has more customers which are sought by 2 and 3 . Note that all providers receive the same payoff when they do not cooperate. Now, if they form the grand coalition and share the aggregate payoff as per an imputation picked randomly from the core, provider 1 is likely to receive the largest share. This is in agreement with our intuitive observation. Another important issue to be pointed out is that, the providers' share of the aggregate payoff is usually largely determined by parameters other than their decision variables. For instance, the number of customers here is not a decision variable and yet it is critical in determining the payoff shares.

Remark: A provider can decide how to upgrade its resources, based on the above observation. For instance, in example III.2, if provider 2 can somehow increase its customer base, its share increases, although the aggregate payoff remains the same. This could be a direction for future work.

\section{BASE STATION GAME}

We now examine cooperation in a setup where providers can decide which channels to rent and where to open base stations. More specifically, a provider $i$ needs to choose a subset of candidate locations $B_{i}$ for the base stations it is going to open. Let $f_{k}$ be the cost of opening base station $k$. Let $b_{k}=1$ if base station $k$ is open and 0 otherwise. Also, $i$ should determine which channels, base station $k \in B_{i}$ will have access to, which are selected from the set $C_{k}$. Once the provider pays the corresponding channel membership costs (i.e., the channel is open), the base station is allowed to use those channels to serve the customers anytime during the operation. Let $g_{l}$ be the membership-based charge of using channel $l \in C_{k}$. Let $c_{l}=1$ if base station $k$ is allowed to use channel $l$ and 0 otherwise. If $c_{l}=1$, the associated provider has to pay the corresponding membership fee, $g_{l}$, a constant number, plus usage-based charges which was discussed before. A channel $l \in C_{k}$, for some $k \in B_{i}$, can serve customer $j$ if a) Base station $k$ is open, b) Channel $l$ is open, and c)Base station $k$ and customer $j$ are associated with the same provider or the providers associated with them are in a coalition. We assume $m_{j}=0$ for all $j \in M_{N}$. Note that, $b_{k}$ and $c_{l}$ are deterministic variables and cannot depend on $\omega$, in contrast to $\alpha_{l j}(\omega) \mathrm{s}$ that are decided to best suit the respective outcome $\omega \in \Omega$. We assume that all utility and cost functions are linear, and $u_{l j}(\omega) \in \mathbb{R}$ is the payment of customer $j$ minus the usagebased charges of channel $l$, per unit of time of service. Now we have the tools to formulate the optimization which provides $v(S)$ for all $S \subseteq N$.

$\mathbf{P}_{\mathrm{G}}(\mathbf{S}):-\mathbf{M a x}: \sum_{\substack{l \in C_{S} \\ j \in M_{S} \\ \omega \in \Omega}} \alpha_{l j}(\omega) u_{l j}(\omega) P(\omega)-\sum_{k \in B_{S}} f_{k} b_{k}-$

$\sum_{l \in C_{S}} g_{l} c_{l}$

Subject to:

1) $\sum_{l \in C_{S}} \alpha_{l j}(\omega) \leq 1, \quad j \in M_{S}, \omega \in \Omega$

2) $\sum_{j \in M_{S}} \alpha_{l j}(\omega) \leq c_{l}, \quad l \in C_{S}, \omega \in \Omega$

3) $c_{l} \leq b_{k}, \quad l \in C_{k}, k \in B_{S}$

4) $\alpha_{l j}(\omega) \in[0, \alpha], \quad l \in C_{S}, j \in M_{S}, \omega \in \Omega$

5) $b_{k}, c_{l} \in\{0,1\}, \quad l \in C_{S}, k \in B_{S}$

Constraints (1) ensure that the total fraction of time a customer is being served, is upper bounded by 1 . If a channel is open, the the fraction of time it can serve is at most 1 , and it cannot serve otherwise, by constraints (2). Constraints (3) guarantee that only opened base stations can have open channels. Unless mentioned otherwise we assume $\alpha=1$ which renders constraints (4) trivial. We discuss the function of constraints (4) for $\alpha<1$ in Subsection IV-C .

We now elucidate the impact of cooperation on the total payoff, as well as the providers' decision variables, using the following example.

Example IV.1. Consider the setup in figure 1 with $B_{1}=$ $C_{1}=\{1\}$ and $B_{2}=C_{2}=\{2,3\}$, where $g_{l}=0$ for $l \in C_{N}$, $f_{1}=0$, and $f_{2}=f_{3}=f$. Let $R_{11}=R_{21}=R_{23}=Q$, $R_{12}=R_{22}=R_{33}=P$, and $R_{l j}=0$ otherwise. Let $m_{j}=0$ for $j=1 \ldots 3, f<P$, and $Q<P$. Let payoffs consist of the sum of the service rates. Now $v(\{1\})=Q$. Also $v(\{2\})=$ $\max [P-f, 2 P-2 f]$ and $v(\{1,2\})=\max [2 P-f, 2 P+$ $Q-2 f]$, where the former payoffs are the result of opening just base station 3, while the latter ones occur when both are open. Intuitively, if provider 2 cooperates with 1 , opening base station 2 may not be necessary. In fact if $Q<f<P$, opening both base stations 2 and 3 is optimal when not in coalition, 
while opening just base station 3 is optimal under cooperation. This is in agreement with the intuition that opening a base station in the area that is covered by other base stations might be redundant. However, if there is a relatively large traffic demand in that area, opening the base station could become optimal. For instance, in our example, if $R_{21}=P, v(\{1,2\})=$ $\max [2 P-f, 3 P-2 f]$ and it is optimal to open both base stations.

Note that the same approach taken in section III to show that the core is nonempty, is inadequate here. The reason is that, this optimization problem involves integer variables and therefore strong duality does not hold in general. However, there are important special cases where similar results extend.

\section{A. Case I}

Consider the special case where customers do not move and the quality of the channels do not vary with time, i.e. $|\Omega|=1$. We also assume that an open base station is allowed to use only one given channel, which has no membership fee, i.e. $C_{k}=\{k\}$ for all $k \in B_{N}$, and $c_{l}=0$ for all $l \in C_{N}$. Then $\mathrm{P}_{\mathrm{G}}(S)$ will reduce to the following IP:

$\mathbf{P}_{\mathrm{b}}(\mathbf{S})$ :- Maximize: $\sum_{\substack{k \in B_{S} \\ j \in M_{S}}} \alpha_{k j} u_{k j}-\sum_{k \in B_{S}} f_{k} b_{k}$

\section{Subject to:}

1) $\sum_{k \in B_{S}} \alpha_{k j} \leq 1, \quad j \in M_{S}$

2) $\sum_{j \in M_{S}} \alpha_{k j} \leq b_{k}, \quad k \in B_{S}$

3) $\alpha_{k j} \geq 0, \quad k \in B_{S}, j \in M_{S}$

4) $b_{k} \in\{0,1\}, \quad k \in B_{S}$

In the following we proceed to prove that the core of the coalitional game $\langle N, v\rangle$, with characteristic function $v($. given by $\mathrm{P}_{\mathrm{b}}(S)$, is nonempty. The proof consists of three steps.

Step I) Consider the coalitional game $\langle N, \hat{v}\rangle$, where $N$ is the same set of providers and the characteristic function $\hat{v}($.$) is$ given by the LP, $\mathrm{P}_{\text {relaxed }}(S)$. $\mathrm{P}_{\text {relaxed }}(S)$ is the linear relaxation of $\mathrm{P}_{\mathrm{b}}(S)$, where the constraints $b_{k} \in\{0,1\}$ are now replaced by $b_{k} \in[0,1]$. We show that the core of the coalitional game $<N, \hat{v}>, \hat{\mathcal{C}}$, is nonempty.

Using $\lambda \in \mathbb{R}^{\left|M_{S}\right|}$, and $\nu, \gamma \in \mathbb{R}^{\left|B_{S}\right|}$, we construct the following LP as the dual of $\mathrm{P}_{\text {relaxed }}(S)$

$\mathbf{D}_{\text {relaxed }}(\mathbf{S})$ :- Minimize: $\sum_{j \in M_{N}} \lambda_{j}+\sum_{k \in B_{N}} \gamma_{k}$

\section{Subject to:}

1) $\lambda_{j}+\nu_{k} \geq u_{k j}, \quad k \in B_{N}, j \in M_{N}$

2) $\nu_{k}-\gamma_{k} \leq f_{k}, \quad k \in B_{N}$

3) $\lambda_{j}, \nu_{k}, \gamma_{k} \geq 0, \quad k \in B_{N}, j \in M_{N}$

Let $\mathcal{D}_{\text {relaxed }}$ constitute the set of optimal solutions of $\mathrm{D}_{\text {relaxed }}(N)$. Define: $\mathcal{I}_{\mathrm{b}}=\left\{\mathrm{x}^{*} \in \mathbb{R}^{|N|}: x_{i}^{*}=\sum_{j \in M_{i}} \lambda_{j}^{*}+\right.$ $\sum_{k \in B_{i}} \gamma_{k}^{*}$ for some $\left.\left(\lambda^{*}, \nu^{*}, \beta^{*}, \gamma^{*}\right) \in \mathcal{D}_{\text {relaxed }}\right\}$.

Theorem IV.1. $\mathcal{I}_{b} \neq \emptyset$, and $\mathcal{I}_{b} \subseteq \hat{\mathcal{C}}$

Proof: The proof is the same as that presented in the proof of theorem III.1.

Step II) In the following we prove that If $\mathrm{P}_{\mathrm{b}}(N)$ has zero integrality gap (i.e., $\mathrm{P}_{\text {relaxed }}(N)$ has an integral optimal solution), the core of $\langle N, v\rangle, \mathcal{C}$, includes the core of the coalitional game $\langle N, \hat{v}\rangle$, and consequently $\mathcal{I}_{\mathrm{b}} \subseteq \mathcal{C}$.

Theorem IV.2. If $\mathrm{P}_{\mathrm{b}}(N)$ has zero integrality gap, i.e. $v(N)=$ $\hat{v}(N)$, then $\hat{\mathcal{C}} \subseteq \mathcal{C}$.
Proof: Consider an arbitrary imputation $\mathbf{x} \in \hat{\mathcal{C}}$. We show that $\mathrm{x} \in \mathcal{C}$. We need to check the two properties of a core element. First note that, by the hypothesis, $v(N)=\hat{v}(N)=$ $x(N)$. Second, $\mathbf{x} \in \hat{\mathcal{C}}$ implies that $x(S) \geq \hat{v}(S), S \subset N$, and because any feasible solution of $\mathrm{P}_{\mathrm{b}}(S)$ is feasible for $\mathrm{P}_{\text {relaxed }}(S)$, we have $\hat{v}(S) \geq v(S), S \subset N$. Thus, $x(S) \geq$ $v(S), S \subset N$. Hence, $\mathbf{x} \in \mathcal{C}$ and the lemma follows.

Step III) Finally, we prove in technical report [4] that $\mathrm{P}_{\mathrm{b}}(N)$ has zero integrality gap.

Here is the main result

Theorem IV.3. $\mathcal{I}_{b} \neq \emptyset$, and $\mathcal{I}_{b} \subseteq \mathcal{C}$

Proof: Using theorems IV.1, IV.2, and the fact that $v(N)=\hat{v}(N)$, the claim immediately follows.

\section{B. Case II}

Consider the case, where now opposite to that in Subsection IV-A, $f_{k}=0$ for all $k \in B_{N}$ and channels can have membership costs. Each base station can now rent more than one channel. Also, $|\Omega|=1$. Under these assumptions, $\mathrm{P}_{\mathrm{G}}(S)$ reduces to an IP with the exact same structure as $\mathrm{P}_{\mathrm{b}}(S)$. Thus, all the formulations and results extend.

\section{Case III}

We consider another special case. Suppose that in order to maintain a certain degree of fairness in the network, providers impose an upper bound on the scheduling decision variables, that is $\alpha_{l j}(\omega) \leq \alpha$ for all $l \in C_{N}, j \in M_{N}, \omega \in \Omega$, for an $\alpha$ such that $\alpha .\left|C_{N}\right| \leq 1$ (Hence constraints (4) in $\mathrm{P}_{\mathrm{G}}(\mathrm{S})$ ). With this assumption, the characteristic function $v_{\mathrm{f}}($.$) , is then$ given by an optimization problem $\mathrm{P}_{\mathrm{f}}(S)$ derived by omitting constraints (1) in $\mathrm{P}_{\mathrm{G}}(S)$. In the following, we proceed to show that the core of the coalitional game $\left\langle N, v_{\mathrm{f}}\right\rangle, \mathcal{C}_{\mathrm{f}}$, is nonempty.

Let $\tau \in \mathbb{R}^{\left|C_{S} \times \Omega\right|}$ and $\varphi \in \mathbb{R}^{\left|C_{S}\right|}$. Define ${ }^{3}$

$h_{k}^{S}(\tau, \varphi)=\max _{\alpha_{l j} \in[0, \alpha], b_{k}, c_{l} \in\{0,1\}}\left(-f_{k} b_{k}-\right.$ $\sum_{l \in C_{k}} g_{l} c_{l}+\sum_{l \in C_{k} j \in M_{S}} \alpha_{l j}(\omega) u_{l j}(\omega) P(\omega)-$ $\left.\sum_{\substack{l \in C_{k} \\ \omega \in \Omega}} \tau_{l}(\omega)\left(\sum_{\substack{l \in C_{k} \\ j \in M_{S}}} \alpha_{l j}(\omega)^{\omega \in \Omega}-c_{l}\right)^{+}-\sum_{l \in C_{k}} \varphi_{l}\left(c_{l}-b_{k}\right)^{+}\right)$

D $_{\mathrm{f}}(S)$ : Minimize: $\sum_{k \in B_{S}} h_{k}^{S}(\tau, \varphi)$

Subject to: $\tau, \varphi \geq 0$

Formulate $\mathrm{D}_{\mathrm{f}}(N)$ by defining vectors $\tau$ and $\varphi$ appropriately. Let $\mathcal{D}_{\mathrm{f}}$ constitute the set of optimal solutions of $\mathrm{D}_{\mathrm{f}}(N)$. Note that $\mathcal{D}_{\mathrm{f}} \neq \emptyset$. Now let $\mathcal{I}_{\mathrm{f}}=\left\{\mathbf{x}^{*} \in \mathbb{R}^{|N|}: x_{i}^{*}=\right.$ $\sum_{k \in B_{i}} h_{k}^{N}\left(\tau^{*}, \varphi^{*}\right)$, for some $\left.\left(\tau^{*}, \varphi^{*}\right) \in \mathcal{D}_{\mathrm{f}}\right\}$.

Theorem IV.4. $\mathcal{I}_{\mathrm{f}} \neq \emptyset$, and $\mathcal{I}_{\mathrm{f}} \subseteq \mathcal{C}_{\mathrm{f}}$.

We prove this theorem in technical report [4] using strong duality for the extended dual problem.

\section{COOPERATION IN MULTI-HOP NETWORKS}

We now study cooperation among providers in multi-hop networks. Intuitively, cooperation in multi-hop networks has all the advantages of that in single hop ones, which is sharing the base stations and spectrum. In addition, it has another benefit which we call power sharing. That is, when the providers cooperate, they can redirect their traffic through possibly better

$$
{ }^{3} A^{+} \triangleq \max [A, 0]
$$


multi-hop routes, which in turn could reduce their transmission power consumption. In this section, we generalize our model to incorporate multi-hop networks. Subsequently, we examine the spectrum pooling coalitional game with transferable payoff in this model and show that the core of the game is nonempty.

Consider a network, where a provider deploys a set of base stations (which might include mobile base stations), to serve a set of customers. Customers communicate with base stations via potentially multi-hop routes, that is, customers can relay packets of other customers. However, when a customer relays others' packets, it uses power without transmitting its own packets. In order to motivate customers, providers agree to discount their charges based on how much they relay. Nevertheless, a customer might want to have a maximum relaying agreement with its provider. In this type of networks, providers must decide the communication routes as well as the allocation of base stations. If now a group of providers agree to cooperate by pooling their base stations and customers, not only can they benefit from sharing other's base stations, but they also enjoy a larger set of relay nodes. This, in turn, can increase the capacity of the network, as well as its power efficiency ${ }^{4}$. Therefore, cooperation in multi-hop networks has even higher potential than that in a single hop network. We now present a framework that captures all these issues.

Let $N$ be the set of providers. Let $B_{i}$ and $M_{i}$ be the sets of provider $i$ 's base stations and customers respectively. We assume that each provider uses its base stations to serve the customers through possibly multi-hop routes. We also assume that each customer can communicate with all base stations and customers. However, the transmission rates depend on the quality of the channels and the source-destination distances, which could be random. Each customer can either communicate directly with base stations, or act as a relay and transmit packets to other customers. Then the service rate of a customer $j$ is defined as the total rate at which $j$ 's packets are delivered to any base station, either via a single or multi-hop route ${ }^{5}$. Let $m_{j}$ be the minimum service rate requirement of customer $j$.

Suppose customer $j$ can transmit to a base station or another customer $l$ at a rate equal to $R_{j l}$, a random variable which is a function of the quality of the channel and location of $j$ and $l$. Let $\Omega$ be the state space of the channel qualities and customer locations. We assume $|\Omega|$ is finite. Let $\omega$ be an outcome of this state space and $\mathbb{P}(\omega)$ be its probability.

Let $S \subseteq N$ be a coalition of providers. Suppose, a base station $k$ and a customer $j$, or two customers, can communicate only when both are associated with the same provider or the providers associated with them are in a coalition. Let random variable $\beta_{j_{1} l}^{j_{2}} \in[0,1]$ be the fraction of time, customer $j_{1}$ transmits packets of customer $j_{2}$, to base station or customer $l$. Without loss of generality we can assume that $\beta_{j_{1} l}^{j_{2}}=0$ for $l=j_{1}$ or $l=j_{2} \cdot \beta_{j_{1} l}^{j_{2}} \mathrm{~s}$ are determined by the allocation scheme.

We assume that base stations in the same area use different

\footnotetext{
${ }^{4}$ Note that for certain customers, the increase in the power usage may not be proportional to that in their service rates, but cooperation increases the power efficiency of the network as a whole.

${ }^{5}$ We consider downlink communications. The model and results can easily be extended to the case where communications involve both uplinks downlinks.
}

channels, and thus their communications do not interfere with each other. Also, suppose that customers and base stations cannot transmit or receive through multiple channels simultaneously, or transmit and receive at the same time. Therefore, the necessary and sufficient condition for the simultaneous transmissions to be successful is that the set of transmitterreceiver pairs form a matching. Similar transmission models have extensively been assumed in related contexts [6], [7]. Using a result in graph theory, a sufficient condition for feasibility of a set of $\beta_{j_{1} l}^{j_{2}} \mathrm{~s}$ is that the fraction of time each customer or base station is communicating be upper bounded by $\theta$, where $\theta$ is a constant between 0 and 1 which depends on the topology of the network [8]. For instance $\theta=1$ for bipartite networks and $\frac{2}{3}$ for general networks. We assume this to be also a necessary condition for feasibility of a scheduling.

We now discuss the mechanism which determines the payoffs providers receive and the costs they incur from serving the customers. Let $T_{j}$ be the maximum fraction of time customer $j$ relays. When the provider associated with customer $j_{1}$ is in coalition $S, j_{1}$ receives a service rate equal to the random variable $y_{j_{1}}(\omega)=\sum_{k \in B_{S}, j_{2} \in M_{S}} R_{j_{2} k}(\omega) \beta_{j_{2} k}^{j_{1}}(\omega)$. Besides, $j_{1}$ relays the traffic for $t_{j_{1}}$ fraction of time, where $t_{j_{1}}(\omega)=\sum_{j_{2}, j_{3} \in M_{S}, k \in B_{S}}\left(\beta_{j_{3} j_{1}}^{j_{2}}(\omega)+\beta_{j_{1} j_{3}}^{j_{2}}(\omega)+\beta_{j_{1} k}^{j_{2}}(\omega)\right)$. Suppose when a node $j$ receives a service rate $y_{j}$ and relays traffic $t_{j}$ fraction of time, it pays the associated provider, an amount of $U_{j}\left(y_{j}, t_{j}\right)$, where $U_{j}(y, t)$ is a concave function increasing in $y$ and decreasing in $t$. Let random variables $p_{j l}(\omega)$ represent the power usage per unit time of transmission of customer $j$ to base station or customer $l$. Then a customer $j_{1}$ in a coalition $S$, has a total power usage of $z_{j_{1}}(\omega)=\sum_{l \in B_{S} \cup M_{S}, j_{2} \in M_{S}} \beta_{j_{1} l}^{j_{2}}(\omega) p_{j_{1} l}(\omega)$. This in turn inflicts a cost equal to $V_{j}\left(z_{j}\right)$, where $V_{j}($.$) is an increasing$ convex function.

The net aggregate payoff available to providers in a coalition is the difference between their utilities and costs. Therefore, in order to maximize their aggregate payoff, providers in a coalition $S$ must decide the routes along which they communicate with each node, and schedule the base stations to those routes based on the position of customers, and payoff and cost functions, subject to minimum rate and maximum relaying constraints. Let $v(S)$ represent the maximum aggregate payoff available to a coalition $S$. Then, $v(S)$ is the optimal value of the objective function of the following convex optimization:

$\mathbf{P}_{\mathrm{m}}(\boldsymbol{S}): \quad$ Maximize: $\quad \sum_{\substack{\omega \in \Omega \\ j \in M_{S}}} \mathbb{P}(\omega) U_{j}\left(y_{j}(\omega), t_{j}(\omega)\right) \quad-$ $\sum_{\substack{\omega \in \Omega \\ j \in M_{S}}} \mathbb{P}(\omega) V_{j}\left(z_{j}(\omega)\right)$

Subject to:

1) $y_{j_{1}}(\omega)=\sum_{\substack{k \in B_{S} \\ j_{2} \in M_{S}}} R_{j_{2} k}(\omega) \beta_{j_{2} k}^{j_{1}}(\omega), j_{1} \in M_{S}, \omega \in \Omega$.

2) $t_{j_{1}}(\omega)=\sum_{j_{2}, j_{3} \in M_{S} \backslash j_{1}}\left(\beta_{j_{3} j_{1}}^{j_{2}}(\omega)+\beta_{j_{1} j_{3}}^{j_{2}}(\omega)+\right.$ $\left.\beta_{j_{1} k}^{j_{2}}(\omega)\right), j_{1} \in M_{S}, \omega \in \Omega$.

3) $z_{j_{1}}(\omega)=\sum_{\substack{l \in B_{S} \cup M_{S} \\ j_{2} \in M_{S}}} \beta_{j_{1} l}^{j_{2}}(\omega) p_{j_{1} l}(\omega), j_{1} \in M_{S}, \omega \in \Omega$.

4) $\sum_{j_{3} \in M_{S}}\left(\beta_{j_{1} j_{3}}^{j_{2}}(\omega) R_{j_{1} j_{3}}(\omega)+\beta_{j_{3} j_{1}}^{j_{2}}(\omega) R_{j_{3} j_{1}}(\omega)\right)=$ $\sum_{k \in B_{S}} \beta_{j_{1} k}^{j_{2}}(\omega) R_{j_{1} k}, j_{1} \neq j_{2} \in M_{S}, \omega \in \Omega$.

5) $t_{j}(\omega)+\sum_{l \in B_{S} \cup M_{S}} \beta_{j l}^{j}(\omega) \leq \theta, j \in M_{S}, \omega \in \Omega$. 
6) $\sum_{j \in M_{S}} \mathbb{P}(\omega) y_{j}(\omega) \geq m_{j}, j \in M_{S}$.

7) $t_{j}(\omega) \leq T_{j}, \quad j \in M_{S}, \omega \in \Omega$.

8) $\beta_{j_{1} l}^{j_{2}}(\omega) \geq 0, \quad j_{1}, j_{2} \in M_{S}, l \in B_{S} \cup M_{S}, \omega \in \Omega$

Constraints 4 ensure that the set of $\beta_{j_{1}}^{j_{2}} \mathrm{~s}$ satisfy the flow feasibility constraints, while constraints 5 guarantee that they constitute a feasible allocation. Constraints 6 and 7 impose minimum service and maximum relaying guarantees, respectively.

We can now form the dual problem and define a set $\mathcal{I}$ the same as in section III. Theorem III.1 extends.

\section{NONTRANSFERABLE PAYOFF GAME}

We have so far discussed cooperation among providers, using coalitional games with transferable payoff framework. We assumed that the providers can share the aggregate payoff. This may not always be the case. For instance, providers might not have a unique method of cost or payoff appraisal agreed on, or even if they have, it may not be possible to confirm their income reports. Also, a provider's satisfaction could depend on factors that cannot be converted to monetary units, and thus cannot be shared with others. Under these circumstances, the payoff of each provider could only consist of whatever it receives from its own subscribers reduced by its own operation costs. Nevertheless, cooperation among providers may still be beneficial.

Example VI.1. Consider example III.1, where now the payoff of each provider is the service rate of its own customer. Let $Q<P<2 Q$. Consider the allocation: $\alpha_{11}=\frac{3 P-Q}{4 P}, \alpha_{12}=$ $\frac{Q+P}{4 P}, \alpha_{21}=\frac{Q}{2 P}, \alpha_{22}=\frac{P-2 Q}{2 P}$, and $\alpha_{23}=\frac{1}{2}$. Then the payoffs are $x_{1}=\frac{Q(5 P-Q)}{4 P}>v(\{1\})$ and $x_{2}=\frac{3 P+Q}{4}>v(\{2\})$. Thus cooperation between the two providers is beneficial. Now consider the optimum allocation that maximized the aggregate payoff in example III.1. Then, $x_{1}=\frac{P}{2}$ and $x_{2}=P+\frac{Q}{2}$. Note that, $x_{1}<v(\{1\})=Q$. Thus in nontransferable payoff framework, the allocation that maximizes the aggregate payoff may not motivate the providers to cooperate.

In these cases, we need to resort to the theory of nontransferable payoff cooperative games to develop a framework for cooperation among providers. In doing so, we first take a short detour to the basic theory of nontransferable payoff games.

\section{A. Nontransferable payoff cooperative games}

Let $\mathcal{N}=\{1, \ldots, n\}$ be the set of players. A coalition $\mathcal{S} \subseteq$ $\mathcal{N}$ is a subset of players who cooperate. Let $A(\mathcal{S})$ be the joint action space of the players in coalition $\mathcal{S}$. Each joint action $a \in A(\mathcal{S})$ leads to a payoff vector $\mathbf{x} \in \mathbb{R}^{|\mathcal{S}|}$. Each coalition $\mathcal{S}$ has an associated set $v(\mathcal{S})$ defined as $v(\mathcal{S})=\left\{\mathbf{x} \in \mathbb{R}^{|\mathcal{S}|}\right.$ : $\exists \mathbf{y} \in \mathbb{R}^{|\mathcal{S}|}, \mathbf{y} \geq \mathbf{x}, \mathbf{y}$ corresponds to some $\left.a \in A(\mathcal{S})\right\}$. [9].

Now the stage is set to introduce the following definition

Definition VI.1. A non transferable payoff cooperative game is a pair $(\mathcal{N}, V)$, where $\mathcal{N}=\{1, \ldots, n\}$ is the set of players, and $V=\left(v(\mathcal{S}) \in \mathbb{R}^{|\mathcal{S}|}, \mathcal{S} \subseteq \mathcal{N}\right)$ is a family of sets satisfying

1) For each $\mathcal{S}, v(\mathcal{S})$ is a closed set.

2) If $\mathbf{y} \in v(\mathcal{S})$ and $\mathbf{x} \in \mathbb{R}^{|\mathcal{S}|}$ with $\mathbf{x} \leq \mathbf{y}$, then $\mathbf{x} \in v(\mathcal{S})$.
3) The set of vectors in $v(\mathcal{S})$ in which each player in $\mathcal{S}$ receives no less than the maximum that he can obtain by himself is a nonempty, bounded set.

Any $\mathbf{x} \in v(\mathcal{N})$ is called a feasible payoff profile. The core $\mathcal{C}$ of the game is the set of feasible payoffs, which can not be blocked by any coalition. Formally,

$\mathcal{C}=\left\{\mathbf{x} \in v(\mathcal{N}): \forall \mathcal{S}, \nexists \mathbf{y} \in v(\mathcal{S})\right.$ such that $\left.\mathbf{y}_{i}>\mathbf{x}_{i} \forall i \in \mathcal{S}\right\}$

In example VI.1, for instance, the pair $\left(\frac{Q(5 P-Q)}{4 P}, \frac{3 P+Q}{4}\right)$ is indeed in the core, since it is not blocked by any coalition. The core in nontransferable payoff cooperative games stabilizes the grand coalition, similar to its transferable payoff counterpart (section II).

\section{B. Nonemptiness of the core of spectrum pooling game}

Consider a spectrum pooling game (section III) with the assumption that customers do not move and the quality of channels do not change with time, i.e. $|\Omega|=1$. Now, let providers form a coalition $\mathcal{S}$. A joint action of the providers will be of the form $\left\{\alpha_{l j}, l \in C_{\mathcal{S}}, j \in M_{\mathcal{S}}\right\}$, which must satisfy the following feasibility constraints.

1) $\sum_{j \in M_{\mathcal{S}}} \alpha_{l j} \leq 1, l \in C_{\mathcal{S}}$.

2) $\sum_{l \in C_{\mathcal{S}}} \alpha_{l j} \leq 1, j \in M_{\mathcal{S}}$.

3) $\sum_{l \in C_{\mathcal{S}}} \alpha_{l j} R_{l j} \geq m_{j}, j \in M_{\mathcal{S}}$.

4) $\alpha_{l j} \geq 0, l \in C_{\mathcal{S}}, j \in M_{\mathcal{S}}$.

Suppose that joint (feasible) action space is nonempty. Customer $j$ associated with provider $i$ receives rate $y_{j}=$ $\sum_{l \in C_{\mathcal{S}}} \alpha_{l j} R_{l j}$ and channel $l$ is used $z_{l}=\sum_{j \in M_{S}} \alpha_{l j}$ fraction of time. Let $\mathbf{y}_{i}=\left\{y_{j}, j \in M_{i}\right\}$ and $\mathbf{z}_{i}=\left\{z_{l}, l \in C_{i}\right\}$. Then, the corresponding payoff vector will be $\left(U_{i}\left(\mathbf{y}_{i}\right)-V_{i}\left(\mathbf{z}_{i}\right), i \in\right.$ $\mathcal{S})$. Now, we show that the core of this game is nonempty. We need the following definitions.

Definition VI.2. A collection (of coalitions) $\mathcal{T} \subset 2^{\mathcal{N}} \backslash \emptyset$ is called balanced if there exist non-negative weights $\left(\lambda_{\mathcal{S}}, \mathcal{S} \in\right.$ $\mathcal{T})$ such that $\sum_{\mathcal{S} \in \mathcal{T}: i \in \mathcal{S}} \lambda_{\mathcal{S}}=1, \forall i \in \mathcal{N}$.

Definition VI.3. A game is balanced if for every balanced collection $\mathcal{T}$, if $u \in \mathbb{R}^{n}$ and $u^{\mathcal{S}} \in v(\mathcal{S})$ for all $\mathcal{S} \in \mathcal{T}$, then $u \in v(\mathcal{N}){ }^{6}$

Finally, we will make use of the following theorem [9].

Theorem VI.1. A balanced game always has a nonempty core.

Here is the main result.

Theorem VI.2. The non transferable payoff spectrum pooling game described above, is balanced and hence has a nonempty core.

Proof: Consider a balanced collection of coalitions $\mathcal{T}$. Let $\left(\lambda_{\mathcal{S}}, \mathcal{S} \in \mathcal{T}\right)$ be the corresponding non-negative weights. Also, let $u \in \mathbb{R}^{n}$ be such that $u^{\mathcal{S}} \in v(\mathcal{S})$ for all $\mathcal{S} \in \mathcal{T}$, i.e, there exist joint actions $\left\{\alpha_{l j}^{\mathcal{S}}, l \in C_{\mathcal{S}}, j \in M_{\mathcal{S}}\right\}$ for all $\mathcal{S} \in \mathcal{T}$ that satisfy constraints $1-4$ and $u_{i} \leq U_{i}\left(\mathbf{y}_{i}^{\mathcal{S}}\right)-V_{i}\left(\mathbf{z}_{i}^{\mathcal{S}}\right), \forall i \in \mathcal{S}$ where $\left\{\mathbf{y}_{i}^{\mathcal{S}}, i \in \mathcal{S}\right\}$, denote the rate vectors corresponding to joint action $\left\{\alpha_{l j}^{\mathcal{S}}, k \in C_{\mathcal{S}}, j \in M_{\mathcal{S}}\right\}$, and $\left\{\mathbf{z}_{i}^{\mathcal{S}}, i \in \mathcal{S}\right\}$ is its corresponding channel utilization vectors. Now, define a joint

\footnotetext{
${ }^{6}$ For any $\mathbf{x} \in \mathbb{R}^{n}, \mathbf{x}^{\mathcal{S}} \in \mathbb{R}^{|\mathcal{S}|}$ is defined by $\mathbf{x}_{i}^{\mathcal{S}}=\mathbf{x}_{i}, \forall i \in \mathcal{S}$.
} 
action $\left\{\alpha_{l j}, l \in C_{\mathcal{N}}, j \in M_{\mathcal{N}}\right\}$ as $\alpha_{l j}=\sum_{\mathcal{S} \in \mathcal{T}: l \in C_{\mathcal{S}}} \lambda_{\mathcal{S}} \alpha_{l j}^{\mathcal{S}}$. The further proof consists of two steps.

Step 1: $\left\{\alpha_{l j}, k \in C_{\mathcal{N}}, j \in M_{\mathcal{N}}\right\}$ satisfy constraints $1-4$, corresponding to the grand coalition $\mathcal{N}$.

$$
\begin{aligned}
\sum_{j \in M_{\mathcal{N}}} \alpha_{l j} & =\sum_{j \in M_{\mathcal{N}}} \sum_{\substack{\mathcal{S} \in \mathcal{T}: l \in C_{\mathcal{S}} \\
j \in M_{\mathcal{S}}}} \lambda_{\mathcal{S}} \alpha_{l j}^{\mathcal{S}} \\
& =\sum_{\mathcal{S} \in \mathcal{T}: l \in C_{\mathcal{S}}} \lambda_{\mathcal{S}} \sum_{j \in M_{\mathcal{S}}} \alpha_{l j}^{\mathcal{S}} \\
& \leq \sum_{\mathcal{S} \in \mathcal{T}: l \in C_{\mathcal{S}}} \lambda_{\mathcal{S}} \\
& =\sum_{\mathcal{S} \in \mathcal{T}: i \in \mathcal{S}} \lambda_{\mathcal{S}}\left(\text { where } l \in C_{i}\right) \\
& =1
\end{aligned}
$$

Similarly, one can show that constraints 2 and 3 are also satisfied. Constraint 4 is trivial.

Step 2: $u_{i} \leq U_{i}\left(\mathbf{y}_{i}\right)-V_{i}\left(\mathbf{z}_{i}\right), \forall i \in \mathcal{N}$ where $\left\{\mathbf{y}_{i}, i \in\right.$ $\mathcal{N}\}$ and $\left\{\mathbf{z}_{i}, i \in \mathcal{N}\right\}$, denote the rate and channel utilization vectors corresponding to joint action $\left\{\alpha_{l j}, k \in C_{\mathcal{N}}, j \in M_{\mathcal{N}}\right\}$, respectively. It is easy to check that the rate received by a customer $j, y_{j}=\sum_{\mathcal{S} \in \mathcal{T}: i \in \mathcal{S}} \lambda_{\mathcal{S}} y_{j}^{\mathcal{S}}$ (where $j \in M_{i}$ ), i.e., for all $j, y_{j}$ is a convex combination of $\left\{y_{j}^{\mathcal{S}}, \mathcal{S} \in \mathcal{T}: i \in \mathcal{S}\right\}$, where $j \in M_{i}$. Similarly, $z_{l}=\sum_{\mathcal{S} \in \mathcal{T}: i \in \mathcal{S}} \lambda_{\mathcal{S}} z_{l}^{\mathcal{S}}$ (where $l \in$ $C_{i}$ ). Since $U_{i}($.$) and V_{i}($.$) are increasing concave and convex$ functions respectively, for each provider $i$,

$$
\begin{aligned}
U_{i}\left(\mathbf{y}^{i}\right)-V_{i}\left(\mathbf{z}^{i}\right) & \geq \sum_{\mathcal{S} \in \mathcal{T}: i \in \mathcal{S}} \lambda_{\mathcal{S}}\left(U_{i}\left(\mathbf{y}_{i}^{\mathcal{S}}\right)-V_{i}\left(\mathbf{z}_{i}^{\mathcal{S}}\right)\right) \\
& \geq \sum_{\mathcal{S} \in \mathcal{T}: i \in \mathcal{S}} \lambda_{\mathcal{S}} u_{i} \\
& =u_{i}
\end{aligned}
$$

From Steps 1 and 2, $u \in v(\mathcal{N})$. Hence, the game is balanced and hence has a nonempty core.

\section{RELATED WORK}

Principles and concepts from cooperative game theory are likely to substantially enrich our understanding of resource allocation in wireless networks, particularly as many resource allocation mechanisms depend on cooperation among nodes. Toward this goal, Nash bargaining solutions have been proposed for power control and spectrum sharing among multiple users [10]. Coalitional games have been used recently for modeling cooperation among nodes in the physical layer [11], [12].

We studied cooperation in higher layers. Previous work in this case was presented in [13] and [14]. The former considered a single-hop network with predetermined locations of base stations, stationary customers, and fixed channel qualities. The latter, on the other hand, studied cooperation in wireless secondary access networks where channel qualities and customer locations change in time according to some known random distribution. The current work generalizes the models and results in [13], [14] in that we examined the problem in which the locations of base stations are part of the decision variables and proved the nonemptiness of the core in important special cases. Furthermore, we considered cooperation in multi-hop networks as well. We also investigated the resource allocation problem in nontransferable payoff cooperative game framework.

We now compare and contrast the proof techniques we used with those used in related papers in cooperative games. The duality technique applied in section III was previously used in [13]-[18]. However, we extended this technique to more general convex and integer optimizations.

\section{REFERENCES}

[1] I. F. Akyildiz, W.-Y. Lee, M. C. Vuran, and S. Mohanty, "Next generation/dynamic spectrum access/cognitive radio wireless networks: a survey," Computer Networks Journal (Elsevier), vol. 50, pp. 2127 2159, September 2006.

[2] M. Osborne and A. Rubinstein, A Course in Game Theory. The MIT press, 1999.

[3] V. Conitzer and T. Sandholm, "Complexity of determining nonemptiness of the core," Technical Reprt CS-02-137, CMU, 2002.

[4] A. Aram, C. Singh, S. Sarkar, and A. Kumar, "Cooperative profit sharing in coalition based resource allocation in wireless networks," in Technical Report, (http://www.seas.upenn.edu/ swati/publication.htm), 2008.

[5] D. Hochbaum and J. Shanthikumar, "Convex separable optimization is not much harder than linear optimization," Journal of the Association for Computing Machinery, vol. 37, pp. 843-862, October 1990.

[6] X. Lin and N. Shroff, "The impact of imperfect scheduling on crosslayer rate control in multihop wireless networks," in Proceedings of INFOCOM, (Miami, Fl), March 2005.

[7] X. Wu and R. Srikant, "Regulated maximal matching: a distributed scheduling algorithm for multihop wireless networks with nodeexclusive spectrum sharing," in Proceedings of IEEE CDC-ECC05, (Seville, Spain), Dec 2005 .

[8] B. Hajek and G. Sasaki, "Link scheduling in polynomial time," IEEE Transactions on Information Theory, vol. 34, no. 5, 1988.

[9] H. E. Scarf, "The core of an n person game," Econometrica, vol. 35, pp. 50-69, January 1967.

[10] J. E. Suris, L. A. DaSilva, Z. Han, and A. B. MacKenzie, "Cooperative game theory for distributed spectrum sharing," in ICC, 2007.

[11] Z. Han and H. V. Poor, "Coalitional games with cooperative transmission: A cure for the curse of boundary nodes in selfish packet-forwarding wireless networks," in 5th International Symposium on Modeling and Optimization in Mobile, Ad Hoc, and Wireless Networks, 2007.

[12] S. Mathur, L. Sankaranarayanan, and N. Mandayam, "Coalitional games in cooperative radio networks," Fortieth Asilomar Conference on Signals, Systems and Computers, pp. 1927-1931, Oct.-Nov. 2006.

[13] S. Sarkar, C. Singh, and A. Kumar, "A coalitional game model for spectrum pooling in wireless data access networks," in Proceedings of 2007 Workshop on Information Theory and Applications (ITA), (UCSD, La Jolla, CA), Jan. 29 - Feb. 22008.

[14] A. Aram, S. Sarkar, C. Singh, and A. Kumar, "A coalitional game framework for cooperative secondary spectrum access," in Proceedings of 46th Annual Allerton Conference on Communication, Control, and Computing, September 2008.

[15] L. S. Shapley and M. Shubik, "The assignment game i: The core," International Journal of Game Theory 1, pp. 111-130, 1972.

[16] J. Sanchez-Soriano, M. A. Lopez, and I. Garcia-Jurado, "On the core of transportation games," Mathematical Social Sciences, vol. 41, pp. 215$225,2001$.

[17] E. Markakis and A. Saberi, "On the core of the multicommodity flow game," Decision support systems, vol. 39, pp. 3-10, 2005.

[18] D. Samet and E. Zamel, "On the core and dual set of linear programming games," Mathematics of Operations Research, vol. 9, pp. 309-316, May 1984. 